\title{
Effect of Childhood Trauma on the Association Between Stress-Related Psychological Factors and Hair Cortisol Level in Young Adults
}

\author{
Won Jae Kim¹, Kyung Mee Park ${ }^{1,2}$, Jung Tak Park ${ }^{3}$, Eunchong Seo ${ }^{1,4}$, \\ Minji Bang ${ }^{1,5}$, Suk Kyoon An ${ }^{1}$, Hye Yoon Park ${ }^{1 凶}$, and Eun Lee ${ }^{1 凶}$ \\ ${ }^{1}$ Department of Psychiatry and Institute of Behavioral Science in Medicine, Yonsei University College of Medicine, Seoul, Republic of Korea \\ 2Department of Hospital Medicine, Yongin Severance Hospital, Yonsei University College of Medicine, Yongin, Republic of Korea \\ ${ }^{3}$ Department of Internal Medicine and Institute of Kidney Disease Research, Yonsei University College of Medicine, Seoul, Republic of Korea \\ ${ }^{4}$ Department of Psychiatry, Veteran Health Service Medical Center, Seoul, Republic of Korea \\ ${ }^{5}$ Department of Psychiatry, CHA Bundang Medical Center, CHA University School of Medicine, Seongnam, Republic of Korea
}

Objective Relationship between hair cortisol concentration (HCC) and stress-related psychological measures are inconclusive, possibly due to overlooked heterogeneity regarding childhood trauma and a lack of comprehensive research on stress-related psychological factors. This study aims to compare young adults without history of childhood trauma to young adults who experienced childhood trauma using HCC and various stress-related psychological factors, as well as investigate the impacts of childhood trauma on the association between HCC and stress-related psychological measures.

Methods A total of 206 young, healthy adults were recruited. We divided participants into two groups depending on whether or not they had suffered moderate-to-severe childhood trauma (CT+ and CT-) and compared HCC and various stress-related psychological measures between groups. Using multiple linear regression analyses, we assessed the associations between HCC and stress-related psychological measures for each group.

Results We found no difference between the groups in HCC or the reported number of stressful life events in the past year; however, $\mathrm{CT}+$ individuals reported higher stress perception, more depressive and anxiety-related symptoms, and more difficulties in emotion regulation than CT- individuals. HCC was associated with emotion dysregulation among the CT- individuals, but not among the CT+ individuals.

Conclusion These findings suggest that history of childhood trauma should be considered in studies using HCC as a biomarker for stress in young adults. Furthermore, HCC might be a useful biomarker of stress and stress-related emotion dysregulation in individuals without moderate-to-severe childhood trauma.

Psychiatry Investig 2021;18(11):1131-1136

Keywords Childhood trauma; Hair cortisol concentration; Stress; Emotion regulation.

\section{INTRODUCTION}

Cortisol levels change when individuals are under stress via the hypothalamus-pituitary-adrenal (HPA) axis. ${ }^{1}$ The hypothalamus secretes vasopressin and corticotropin-releasing hormone when an individual is under stress, which stimulates the pituitary gland to secrete adrenocorticotropic hormone. ${ }^{2}$ This, in turn, stimulates the adrenal cortex to increase the cortisol level, which regulates various systems in the body to help the individual cope with stress. ${ }^{3}$ Cortisol has been measured in serum, saliva, and urine as an objective marker for stress; ${ }^{4}$ however, serum-based and saliva-based methods have limitations,

\footnotetext{
Received: August 3, 2021 Revised: September 15, 2021 Accepted: October 15, 2021

$\triangle$ Correspondence: Hye Yoon Park, MD, PhD

Department of Psychiatry, Yonsei University College of Medicine, 50-1 Yonsei-ro, Seodaemun-gu, Seoul 03722, Republic of Korea

Tel: +82-2-2224-1819, Fax: +82-2-313-0891, E-mail: hyeyoonp@yuhs.ac

$\triangle$ Correspondence: Eun Lee, MD, PhD

Department of Psychiatry, Yonsei University College of Medicine, 50-1 Yonsei-ro, Seodaemun-gu, Seoul 03722, Republic of Korea

Tel: +82-2-2228-1620, Fax: +82-2-313-0891, E-mail: leeeun@yuhs.ac

(c) This is an Open Access article distributed under the terms of the Creative Commons Attribution Non-Commercial License (https://creativecommons.org/licenses/by-nc/4.0) which permits unrestricted non-commercial use, distribution, and reproduction in any medium, provided the original work is properly cited
} 
including diurnal variation and invasive sampling. ${ }^{5}$ In recent years, to overcome these limitations, hair samples have been used to measure human cortisol. Hair cortisol concentration (HCC), which represents the average cortisol concentration of the preceding months, has advantages over saliva-based or plasma-based methods of cortisol analysis because it is less invasive and is unaffected by diurnal variations in cortisol level. ${ }^{5}$ These characteristics make HCC more suitable than other forms of cortisol measurement for psychiatric research on chronic stress.

Despite efforts to use HCC as a biomarker of stress, associations between cortisol concentrations and stress-related psychological factors are inconsistent. ${ }^{6}$ One possible reason for this inconsistency is a lack of consideration of childhood trauma, which affects both stress-related psychological factors ${ }^{7,8}$ and HPA axis function. ${ }^{9-11}$ Childhood trauma has been reported to increase depression and anxiety ${ }^{7}$ and disturb the automatic regulation of emotions later in life. ${ }^{8}$ Disruption of the HPA axis and a blunted cortisol response were also shown to be related to childhood trauma. ${ }^{9-11}$ However, the effect of childhood trauma on HCC is still inconclusive. While one meta-analysis ${ }^{12}$ reported a small positive effect to a robust negative effect, a more recent study with a large adult cohort ${ }^{13}$ reported no difference on HCC. Furthermore, because these previous studies have not considered stress-related psychological factors, the role of childhood trauma in the relationship between psychological factors and HCC is unclear.

Another possible reason for the inconsistent association between cortisol and stress-related psychological factors is that various measures of psychological stress were not analyzed comprehensively. HPA axis and cortisol levels have been shown to respond differently to various psychological factors; while they were not associated with self-reported stress perception, ${ }^{6}$ they were affected by psychological states, such as depression and anxiety ${ }^{14}$ and the ability to regulate emotions. ${ }^{15}$

We examined the impacts of childhood trauma on the relationship between HCC and various stress-related psychological measures in young, healthy adults. We hypothesized that compared with young adults with no history of childhood trauma, young adults with a history of childhood trauma would have more difficulties in stress perception and emotion regulation but would not have a correspondingly elevated HCC due to the negative effects of childhood trauma on the HPA axis. Furthermore, given that people with childhood trauma have exhibited changes in stress-related psychological factors and cortisol levels, we hypothesized that the association between HCC and stress-related psychological measures would be different in individuals with a history of childhood trauma than in individuals without such history.

\section{METHODS}

\section{Participants}

Two hundred six individuals, 105 females and 101 males, ranging in age from 19 to 30 years old were recruited via internet job advertisement or posters at Severance Hospital of the Yonsei University Health System, Seoul, Korea. Participants were assessed using the Structured Clinical Interview in the Diagnostic and Statistical Manual of Mental Disorders IV and found to have no major psychiatric illness. Individuals were excluded if they had history of one or more of the following: 1) neurological illness; 2 ) head trauma accompanied by loss of consciousness; 3) a medical or surgical condition requiring hospitalization; 4) discharge from a hospital in the past six months; 5) use of glucocorticoid medication, oral contraceptives, or hormone replacement therapy; or 6) current pregnancy or breastfeeding. This study was approved by the Institutional Review Boards at Severance Hospital of the Yonsei University Health System, Seoul, Korea (4-2014-0744). Written informed consent was obtained from all participants.

\section{Measures}

\section{Childhood Trauma Questionnaire}

The participants were divided into two groups depending whether or not they had a history of moderate-to-severe childhood trauma as determined by the 28 -item Childhood Trauma Questionnaire (CTQ). ${ }^{16}$ Five categories of childhood trauma were assessed: emotional abuse, physical abuse, sexual abuse, emotional neglect, and physical neglect. Considering past reports of lower level of childhood trauma being relatively common among the general population, ${ }^{17}$ dichotomous cutoff points between 'none-to-minimal' and 'moderate-to-severe' were used for each category of childhood trauma (i.e., scores greater than $12,9,7,14$, and 9 indicating moderate-to-severe emotional abuse, physical abuse, sexual abuse, emotional neglect, and physical neglect, respectively).

\section{Stress-related psychological measures}

Multiple psychological measures for different aspects of stress were assessed. The Korean version of the Social Readjustment Rating Scale (K-SRRS) was used to identify major stressful life events that occurred over the last year. ${ }^{18}$ For each reported stressful event, the participants indicated how many times it occurred in the past 12 months, and the weighted sum over all events was calculated to produce a total score. The Cronbach's alpha measure of the internal consistency of the K-SRRS was 0.717 for the present study. The Perceived Stress Scale (PSS) ${ }^{19}$ and the Global Assessment of Recent Stress (GARS) scale ${ }^{20}$ were used to quantify subjective feelings or thoughts that followed 
after a stressful event or encounter. Although participants with major psychiatric disorders were excluded a priori from the study, the Beck Depression Inventory $(\mathrm{BDI})^{21}$ and the StateTrait Anxiety Inventory (STAI) ${ }^{22}$ were used to assess the participants' emotional states, including depression and anxiety symptoms. The Difficulties in Emotional Regulation Scale (DERS) ${ }^{23}$ was used to investigate the participants' emotion regulation by asking if the participants understand and accept their own emotions and how they behave when they experience negative emotions. Each item in the DERS was scored on a five-point Likert scale, with a higher score indicating trouble accepting or regulating emotions (range: 36-180).

\section{Hair cortisol measurement}

Approximately 10 strands or $10 \mathrm{mg}$ of hair were collected using scissors. To minimize variance due to the hair growth rate, the hair sample was collected from the posterior vertex of the head. ${ }^{24}$ For each strand, the $3 \mathrm{~cm}$ of hair closest to the scalp was used for cortisol measurement. Assuming an average growth rate of $1 \mathrm{~cm}$ of hair per month, the sample was taken to represent the average cortisol level of the preceding three months. The hair samples were stored at room temperature for 12 months in a plastic bag. The hair protein was then extracted using a Minute $^{\mathrm{TM}}$ Protein extraction kit (Invent Biotechnologies, Plymouth, MN, USA). The total amount of protein in each sample was normalized using a Pierce ${ }^{\mathrm{TM}}$ BCA Protein Assay Kit (Thermo Fisher Scientific, Waltham, MA, USA). Enzyme-linked immunosorbent assays were performed to detect the cortisol concentration with a limit of detection of $1.14 \mathrm{ng} / \mathrm{mL}$ (lower $95 \%$ confidence limit). The final value of HCC was recorded as pg hair cortisol/ $\mu \mathrm{g}$ hair protein. The mean intra-assay coefficient of variation was $3.47 \%$.

\section{Statistical analysis.}

All statistical analyses were conducted using SPSS version 25 (IBM Corp., Armonk, NY, USA). To produce a normal distribution of responses, logarithmic transformation was applied to the HCC. HCC had a skewness level that were acceptable for statistical analysis $(<1.0)$ after transformation. Chi-square tests and independent t-tests were used to compare baseline and clinical characteristics between groups. For each group, multiple linear regression models were developed to assess the associations between stress-related psychological measures and HCC. Sex was included as an independent variable in all regression analyses. Each model was built using stepwise forward selection ( $p$-value threshold: $\leq 0.05$ to enter, $\geq 0.10$ to remove). Participants with missing data were not included in the corresponding regression analyses. The significance threshold was $\mathrm{p}<0.05$ for all analyses.

\section{RESULTS}

\section{The effect of childhood trauma on HCC and stress-related measures}

Eighty study participants reported moderate-to-severe childhood trauma $(\mathrm{CT}+)$. All participants were psychotropic drug naive. The results of between-group analyses of baseline characteristics, HCC, and stress-related measures are shown in Table 1. There was no significant difference in HCC or number of stressors between the two groups; however, the CT+ group had significantly worse perceived stress levels (PSS: $t=-2.9, \mathrm{p}=0.005$; GARS: $\mathrm{t}=-2.4, \mathrm{p}=0.016)$, depressive symptoms $(\mathrm{t}=-2.5, \mathrm{p}=0.012)$, anxiety symptoms (Trait Anxiety Inventory: $\mathrm{t}=-2.4, \mathrm{p}=0.017$ ), and difficulties in emotion regulation $(\mathrm{t}=-3.3, \mathrm{p}=0.001)$.

\section{Associations between stress-related psychological measures and HCC}

For the CT- group, emotion dysregulation was significantly associated with $\mathrm{HCC}(\mathrm{B}=0.010, \mathrm{p}=0.018)$ in the regression model $\left(\mathrm{R}^{2}=0.046 ; \mathrm{F}=5.798 ; \mathrm{p}=0.018\right)$ (Table 2$)$. The $\mathrm{R}^{2}$ value of the model was 0.046 , indicating that $4.6 \%$ of the total variance can be explained by our model. For the CT+ group, none of the stress-related psychological measures was significantly associated with HCC.

\section{DISCUSSION}

We investigated the associations between stress-related psychological factors and HCC in a cohort of young adults stratified by history of childhood trauma. Even without major psychiatric illness or stressful events, CT+ group had significant disturbance in stress perception, depressive symptoms, trait anxiety and emotion regulation. However, there was no difference in HCC itself between CT+ and CT- group. HCC was associated with difficulty in emotion regulation among individuals without history of childhood trauma. However, no psychological factors were associated with HCC in the CT+ group, suggesting that the relationship between psychological factors and HCC was different between adults without childhood trauma and adults with childhood trauma. These results underscore the importance of considering childhood trauma when conducting research on psychological stress, especially when cortisol level is used as an indicator of stress.

Compared with the CT- individuals, the CT+ individuals showed disturbances in stress perception, emotional states, and emotion regulation, despite the fact that none of the participants had major psychiatric illness. Previous reports of elevated scores on self-assessments of depression and anxiety ${ }^{7}$ and disruption of emotion regulation ${ }^{8}$ among individuals with history of childhood trauma are in line with our findings. Even in 
Table 1. Baseline characteristics and stress-related psychological measures of the study participants

\begin{tabular}{|c|c|c|c|}
\hline & No childhood trauma $(\mathrm{N}=126)$ & Childhood trauma $(\mathrm{N}=80)$ & p-value ${ }^{\ddagger}$ \\
\hline Sex, male/female & $61 / 65$ & $40 / 40$ & 0.824 \\
\hline Age, years & $23.0(2.7)$ & $23.0(2.5)$ & 0.903 \\
\hline Education*, years & $14.3(1.5)$ & $14.5(1.3)$ & 0.465 \\
\hline \multicolumn{4}{|c|}{ Type of childhood trauma } \\
\hline Emotional abuse & - & 10 & \\
\hline Physical abuse & - & 22 & \\
\hline Sexual abuse & - & 9 & \\
\hline Emotional neglect & - & 54 & \\
\hline Physical neglect & - & 44 & \\
\hline 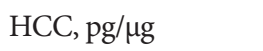 & $7,254.4(8371.7)$ & $8,183.7(9136.1)$ & 0.455 \\
\hline \multicolumn{4}{|c|}{ Stress-related psychological measures } \\
\hline K-SRRS ${ }^{\dagger}$ & $594.6(1027.4)$ & $634.5(1405.1)$ & 0.815 \\
\hline PSS & $24.7(5.6)$ & $27.0(5.6)$ & 0.005 \\
\hline GARS* & $21.6(11.2)$ & $25.6(12.0)$ & 0.016 \\
\hline BDI* & $4.3(4.3)$ & $7.3(9.8)$ & 0.012 \\
\hline SAI & $35.8(9.2)$ & $38.0(8.8)$ & 0.094 \\
\hline TAI & $40.1(6.8)$ & $42.5(7.8)$ & 0.017 \\
\hline DERS & $70.0(16.4)$ & $78.4(19.0)$ & 0.001 \\
\hline
\end{tabular}

Data are presented as the $\mathrm{N}$ or mean (SD). ${ }^{*}$ level of education, GARS, and BDI data were available for 125 participants without childhood trauma and for all participants with childhood trauma; ${ }^{\dagger}$-SRRS data were available for 125 participants without childhood trauma and for

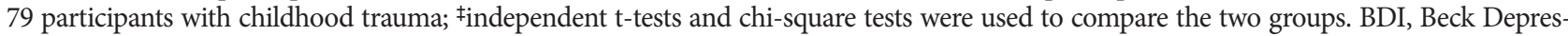
sion Inventory; DERS, Difficulty in Emotion Regulation Scale; GARS, Global Assessment of Recent Stress; HCC, hair cortisol concentration; K-SRRS, Korean version of the Social Readjustment Rating Scale; PSS, Perceived Stress Scale; SAI, State Anxiety Inventory; SD, standard deviation; TAI, Trait Anxiety Inventory

Table 2. Stepwise multiple linear regression of hair cortisol concentration on stress-related psychological measures

\begin{tabular}{|c|c|c|c|c|c|c|c|}
\hline Childhood trauma & Independent variable & $\mathrm{B}$ & SE & $\beta$ & $\mathrm{t}$ & p-value & Model properties \\
\hline \multirow[t]{4}{*}{ Absent $(\mathrm{N}=123)$} & (Constant) & 7.810 & 0.313 & & 24.967 & $<0.001$ & $\mathrm{R}^{2}=0.046$ \\
\hline & DERS & 0.010 & 0.004 & 0.214 & 2.408 & 0.018 & adj. $R^{2}=0.038$ \\
\hline & & & & & & & $\mathrm{F}=5.798$ \\
\hline & & & & & & & $\mathrm{p}=0.018$ \\
\hline Present $(\mathrm{N}=79)$ & No variables were entere & to the ec & & & & & \\
\hline
\end{tabular}

DERS, Difficulty in Emotion Regulation Scale; SE, standard error

the absence of psychiatric illness, young individuals who experienced moderate-to-severe childhood trauma may nonetheless endure turbulent and long-lasting psychological changes.

Difficulty in emotion regulation was a predictor of increased HCC in CT- individuals. Considering that emotion regulation has a moderating effect on stress and HCC, ${ }^{15}$ our findings suggest that screening for difficulties in emotion regulation might be valuable when estimating physiological reactions to stress. On the other hand, subjective stress perception was not associated with HCC. This is in line with a previous report that found no significant associations between HCC and self-reported measures of perceived stress. ${ }^{6}$ Given that the major stressful life events in the past year was also not associated with HCC in our study, the number of stressful events encountered, as well as individuals' subjective perceptions of those events, might not have a major influence on the physiological response to stress. Neither depressed mood nor anxiety was associated with HCC in our regression model. By contrast, a previous study found that depression and anxiety were associated with exaggerated and blunted HPA axis function measured by salivary cortisol responses, respectively. ${ }^{14}$ The contrasting results may indicate that the relationships between psychological symptoms and HCC are moderated by additional unknown factors. Taken altogether, the results suggest that interventions to help individuals cope with uncomfortable emotions might be beneficial in lowering stress, even when perceived stress or psychological 
symptoms of stress are not prominent.

Among the CT+ individuals, none of the stress-related psychological measures was associated with HCC. This lack of association in $\mathrm{CT}+$ individuals could provide explanation to our prior results that showed no difference in HCC between $\mathrm{CT}+$ and CT- group, although there were significant differences in stress-related psychological factors. Chronic exposure to elevated corticotropin-releasing hormone levels has been linked to down-regulation and dysregulation of the HPA axis in adults with history of childhood trauma. ${ }^{25}$ Our findings provide further evidence that the HPA axis function in response to stressrelated psychological changes is reduced in adults with history of childhood trauma. Because the small number of CT+ participants $(n=79)$ in relation to the number of CT- participants $(n=123)$ might have led to false-negative findings, replication of this study with a larger cohort is needed to verify our findings.

Our study has a few limitations. First, we cannot determine causality between the variables because of the cross-sectional nature of data. Hence, future longitudinal studies would be beneficial. Second, although we excluded individuals with severe medical diseases, detailed medical factors and anthropometric factors were not used in the analysis. The effect of those factors would be negligible in young, healthy adults; however, consideration of factors such as inflammatory state or body mass index might eliminate potential confounding effects. Third, there were differences in the time scales of the psychological assessments and the HCC measurement. For example, $3 \mathrm{~cm}$ of hair represents roughly 3 months of cortisol levels, whereas the K-SRRS asks about stressful events over the previous year. Fourth, since hair characteristics have been suggested to be considered as covariates, ${ }^{6}$ including more comprehensive features such as hair washing frequency or hair treatment in the analysis of HCC may further enhance our understanding. Fifth, childhood trauma was reported retrospectively, so recall bias could exist; however, the CTQ is one of the most frequently used methods to measure traumatic childhood experiences. ${ }^{26,27}$ The CTQ was validated using adolescents with corroborative data, ${ }^{16}$ but the results should be interpreted with caution. Finally, we could not investigate each subtype of childhood trauma separately because the sample size for each subtype would have been too small for statistical testing. In future studies, the subtype of childhood trauma could be an important factor to consider in the relationship between childhood trauma and HCC, ${ }^{12}$ and a larger study accounting for each separate category of childhood trauma is warranted for a more thorough analysis.

In conclusion, our findings suggest that HCC might be a useful biomarker for stress-related psychological factors, especially difficulty in emotion regulation, in people without history of moderate-to-severe childhood trauma.

\section{Availability of Data and Material}

The datasets generated or analyzed during the study are available from the corresponding author on reasonable request.

\section{Conflicts of Interest}

Minji Bang, a contributing editor of the Psychiatry Investigation, was not involved in the editorial evaluation or decision to publish this article. All remaining authors have declared no conflicts of interest.

\section{Author Contributions}

Conceptualization: Won Jae Kim, Hye Yoon Park, Eun Lee. Data curation: Kyung Mee Park, Jung Tak Park, Eunchong Seo, Minji Bang, Suk Kyoon An. Formal analysis: Won Jae Kim, Hye Yoon Park. Funding acquisition: Eun Lee. Investigation: Won Jae Kim, Hye Yoon Park. Methodology: Won Jae Kim, Hye Yoon Park, Eun Lee. Project administration: Hye Yoon Park, Eun Lee. Resources: Kyung Mee Park, Jung Tak Park, Eunchong Seo, Minji Bang, Suk Kyoon An. Supervision: Kyung Mee Park, Jung Tak Park, Eunchong Seo, Minji Bang, Suk Kyoon An. Visualization: Won Jae Kim, Hye Yoon Park. Writing_-original draft: Won Jae Kim. Writing_review \& editing: all authors.

\section{ORCID iDs}

Won Jae Kim https://orcid.org/0000-0003-3472-5432

Kyung Mee Park ～https://orcid.org/0000-0002-2416-2683

Jung Tak Park https://orcid.org/0000-0002-2325-8982

Eunchong Seo https://orcid.org/0000-0003-2644-266X

Minji Bang https://orcid.org/0000-0002-1669-4014

Suk Kyoon An $\quad$ https://orcid.org/0000-0003-4576-6184

Hye Yoon Park https://orcid.org/0000-0001-9579-8112

Eun Lee https://orcid.org/0000-0002-7462-0144

\section{Funding Statement}

The National Research Foundation of Korea funded by the Ministry of Science, ICT \& Future Planning, Republic of Korea, supported the present work (Grant number: 2017R1A2B3008214 to Eun Lee).

\section{REFERENCES}

1. Tsigos C, Chrousos GP. Hypothalamic-pituitary-adrenal axis, neuroendocrine factors and stress. J Psychosom Res 2002;53:865-871.

2. Charmandari E, Tsigos C, Chrousos G. Endocrinology of the stress response. Annu Rev Physiol 2005;67:259-284.

3. Chrousos GP. Stress and disorders of the stress system. Nat Rev Endocrinol 2009;5:374-381.

4. Levine A, Zagoory-Sharon O, Feldman R, Lewis JG, Weller A. Measuring cortisol in human psychobiological studies. Physiol Behav 2007; 90:43-53.

5. Koumantarou Malisiova E, Mourikis I, Darviri C, Nicolaides NC, Zervas IM, Papageorgiou C, et al. Hair cortisol concentrations in mental disorders: a systematic review. Physiol Behav 2021;229:113244.

6. Stalder T, Steudte-Schmiedgen S, Alexander N, Klucken T, Vater A, Wichmann S, et al. Stress-related and basic determinants of hair cortisol in humans: a meta-analysis. Psychoneuroendocrinology 2017;77:261274.

7. Mirman A, Bick AS, Kalla C, Canetti L, Segman R, Dan R, et al. The imprint of childhood adversity on emotional processing in high functioning young adults. Hum Brain Mapp 2021;42:615-625.

8. Marusak HA, Martin KR, Etkin A, Thomason ME. Childhood trauma exposure disrupts the automatic regulation of emotional processing. Neuropsychopharmacology 2015;40:1250-1258.

9. Carpenter LL, Shattuck TT, Tyrka AR, Geracioti TD, Price LH. Effect of childhood physical abuse on cortisol stress response. Psychopharmacology (Berl) 2011;214:367-375.

10. O'Connor DB, Green JA, Ferguson E, O'Carroll RE, O'Connor RC. Ef- 
fects of childhood trauma on cortisol levels in suicide attempters and ideators. Psychoneuroendocrinology 2018;88:9-16.

11. Schalinski I, Teicher MH, Rockstroh B. Early neglect is a key determinant of adult hair cortisol concentration and is associated with increased vulnerability to trauma in a transdiagnostic sample. Psychoneuroendocrinology 2019;108:35-42.

12. Khoury JE, Bosquet Enlow M, Plamondon A, Lyons-Ruth K. The association between adversity and hair cortisol levels in humans: a metaanalysis. Psychoneuroendocrinology 2019;103:104-117.

13. Oresta S, Vinkers CH, van Rossum EFC, Penninx B, Nawijn L. How childhood trauma and recent adverse events are related to hair cortisol levels in a large adult cohort. Psychoneuroendocrinology 2021;126: 105150.

14. Fiksdal A, Hanlin L, Kuras Y, Gianferante D, Chen X, Thoma MV, et al. Associations between symptoms of depression and anxiety and cortisol responses to and recovery from acute stress. Psychoneuroendocrinology 2019;102:44-52.

15. Klosowska JC, Verbeken S, Braet C, Wijnant K, Debeuf T, De Henauw $\mathrm{S}$, et al. The moderating role of emotion regulation in the association between stressors with psychological and biological measures in adolescence. Psychosom Med 2020;82:495-507.

16. Bernstein DP, Stein JA, Newcomb MD, Walker E, Pogge D, Ahluvalia $\mathrm{T}$, et al. Development and validation of a brief screening version of the Childhood Trauma Questionnaire. Child Abuse Negl 2003;27:169190.

17. Baker AJ, Maiorino E. Assessments of emotional abuse and neglect with the CTQ: issues and estimates. Child Youth Serv Rev 2010;32:740748 .
18. Hong KE, Jeong DU. Construction of Korean 'social readjustment rating scale'- A methodological study. J Korean Neuropsychiatr Assoc 1982;21:123-136.

19. Cohen S, Kamarck T, Mermelstein R. A global measure of perceived stress. J Health Soc Behav 1983;24:385-396.

20. Linn MW. A Global Assessment of Recent Stress (GARS) Scale. Int J Psychiatry Med 1985;15:47-59.

21. Beck AT, Steer RA. BDI, Beck Depression Inventory: Manual. San Antonio, Tex: Psychological Corporation; 1987.

22. Spielberger C, Gorsuch R, Lushene R, Vagg PR, Jacobs G. Manual for the State-Trait Anxiety Inventory (Form Y1-Y2). Palo Alto, CA: Consulting Psychologists Press; 1983.

23. Gratz KL, Roemer L. Multidimensional assessment of emotion regulation and dysregulation: development, factor structure, and initial validation of the difficulties in emotion regulation scale. J Psychopathol Behav Assess 2004;26:41-54.

24. Pragst F, Balikova MA. State of the art in hair analysis for detection of drug and alcohol abuse. Clin Chim Acta 2006;370:17-49.

25. De Bellis MD, Zisk A. The biological effects of childhood trauma. Child Adolesc Psychiatr Clin N Am 2014;23:185-222, vii.

26. Ford BN, Yolken RH, Aupperle RL, Teague TK, Irwin MR, Paulus MP, et al. Association of early-life stress with cytomegalovirus infection in adults with major depressive disorder. JAMA Psychiatry 2019;76:545547.

27. Opel N, Redlich R, Dohm K, Zaremba D, Goltermann J, Repple J, et al. Mediation of the influence of childhood maltreatment on depression relapse by cortical structure: a 2 -year longitudinal observational study. Lancet Psychiatry 2019;6:318-326. 